\title{
Intensity Modulated Proton Therapy as a Boost Treatment after Prostate Seed Implant: A Treatment Planning Study
}

\author{
Junqing $\mathrm{Wu}^{1}$, Huanmei $\mathrm{Wu}^{2 *}$, Chee-Wai Cheng ${ }^{3}$ \\ ${ }^{1}$ School of Health Sciences, Purdue University, West Lafayette, USA \\ ${ }^{2}$ Purdue School of Engineering and Technology, Indiana University School of Informatics, IUPUI, Indianapolis, USA \\ ${ }^{3}$ Department of Radiation Oncology, Indiana University School of Medicine, Indianapolis, USA \\ Email: wu154@purdue.edu
}

Received 26 January 2015; accepted 9 February 2015; published 15 February 2015

Copyright (C) 2015 by authors and Scientific Research Publishing Inc.

This work is licensed under the Creative Commons Attribution International License (CC BY). http://creativecommons.org/licenses/by/4.0/

(c) (7) Open Access

\begin{abstract}
Purpose: Combination of Prostate Seed Implant (PSI) with External Beam Radiation Therapy (EBRT) remains as an attractive option for patients with intermediate or high-risk prostate cancer. One of the most widely used approaches is to use external beam radiation therapy (EBRT) to deliver boost doses after permanent prostate seed implant (PSI). In this study, the feasibility of using Intensity Modulated Proton Therapy (IMPT) as an alternative EBRT boost treatment for PSI patients was investigated in the presence of a large number of high $\mathrm{Z}$ metallic seeds. The dosimetry of IMPT boost plans was compared with that of conventional Intensity Modulated Radiation Therapy (IMRT) boost plans. Methods: Ten post prostate seed implants with seeds of I-125 were randomly selected for this study. Proton treatment plans were created with two lateral opposed proton beams in Eclipse treatment planning system. IMRT boost plans were generated with seven co-planner beams for comparison. Several plan evaluation parameters such as the planning target volume (PTV) dose homogeneity, dose conformity and dose to surrounding normal tissues were evaluated. Results: Compared to conventional IMRT boost plans, IMPT demonstrated better sparing of normal tissues while providing similar satisfactory PTV coverage. The high $\mathrm{Z}$ implanted seeds is not a problem for IMPT as boost treatment. Conclusions: PSI with an IMPT boost can be a valuable option for prostate cancer patient treatment. It delivers comparable or better radiation dose distribution in terms of normal tissue sparing compared to IMRT boost plan.
\end{abstract}

\section{Keywords}

IMPT, Prostate Seed Implant, IMRT

${ }^{*}$ Corresponding author.

How to cite this paper: Wu, J.Q., Wu, H.M. and Cheng, C.-W. (2015) Intensity Modulated Proton Therapy as a Boost Treatment after Prostate Seed Implant: A Treatment Planning Study. International Journal of Medical Physics, Clinical Engineering and Radiation Oncology, 4, 76-83. http://dx.doi.org/10.4236/ijmpcero.2015.41011 


\section{Introduction}

Combined radiotherapy of prostate seed implant (PSI) with External Beam Radiation Therapy (EBRT) provides an attractive option for patients with intermediate or high-risk prostate disease as it delivers a higher radiobiological dose to the prostate compared to EBRT alone with similar level of anticipated toxicity [1] [2]. The standard approach of combined therapy includes the deliveries of seed implant and external-beam radiotherapy. The order of these two components to be executed could play an important role in term of treatment outcome. If EBRT is followed by PSI, it would be extremely difficult to compensate sub-optimal dose distribution delivered by PSI, if not impossible. On the other hand, PSI followed by EBRT is an attractive combination as the dose irregularities from PSI may be rectified by the subsequent EBRT [3].

Traditional 3-dimensional conformal planning has limited capability for dose correction or dose reduction to surrounding normal organs. For instance, it is very difficult to boost the coverage to a low dose region inside the PTV with 3D conformal radiation therapy. However, the wide implementation of Intensity Modulated Radiation Therapy (IMRT) has been proved to overcome the high dose irregularities produced by prostate seed implant (PSI) [4] [5]. The drawback of IMRT is the increased dose inhomogeneity within the planning target volume, which could potentially increase the incidence of long-term side effects when coupled to the dose inhomogeneity inherited from PSI [6]-[8].

In recent years, Proton Therapy (PT) has seen steadily growing popularity as an emerging external beam radiotherapy technique. Proton centers increased from 3 in 2001 to 13 in 2014 in the U.S. with a few more under construction or in the planning stage. PT is favored because of its sharp dose fall-off beyond the proton Bragg peak and its ability to precisely localize the radiation dosage. Similar to IMRT, intensity modulated proton therapy (IMPT) is a treatment delivery technique based on inverse planning optimization. The treatment planning system (TPS) evaluates the cost function of IMPT plan, adjusts the pencil beam weight, and achieves an optimized delivery plan. Among the IMPT techniques, spot-scanning-based IMPT is the most attractive modality, which modulates the intensity and energy of each small, mono-energetic proton beam spot individually under computer control. Different energy of the protons leads to different pencil beam range and energy spread of the protons determines the sharpness of Bragg Peak. One of the major advantages of spot scanning technique is that beam apertures and field-specific range compensators are not required, resulting in reduced neutron production and higher flexibility for adaptive planning [9].

In this study, the feasibility of IMPT as a boost for I-125 permanent seed implant patients with localized prostate cancer was investigated. The results from IMPT were compared with those of IMRT plans. Several plan evaluation parameters such as the planning treatment volume (PTV) dose homogeneity, dose conformity and dose to surrounding normal tissues were evaluated to determine if IMPT can be a valuable option as a boost for PSI.

\section{Method and Materials}

\subsection{Prostate Seed Implant}

Ten PSI patients were randomly selected and evaluated retrospectively in this study. I-125 prostate seeds were $4.5 \mathrm{~mm}$ long and $0.8 \mathrm{~mm}$ in diameter, manufactured by Bard Medical, Inc (Covington, Georgia), with activities ranging from $0.3 \mathrm{mCi}$ to $0.4 \mathrm{mCi}$. I-125 seed contains a titanium tube ( $0.08 \mathrm{~mm}$ thickness), a copper coating, an aluminum layer and a gold core marker. The seed implantation procedure which was previously described [10] used a real-time intra-operative planning approach with a Varian VariSeed treatment planning system and a prescription dose of $110 \mathrm{~Gy}$. Patients were under general anesthesia during the implantation and ultrasound was used to guide needle placements. The dose criteria used in treatment planning included that prostate $V_{100}$ was larger than $99 \%, V_{150}$ was between $50 \%$ and $65 \%$, and $\mathrm{D}_{90}$ was below $125 \%$ of the prescribed dose.

\subsection{Post-Implant CT Simulation}

The post-implant CT scans were obtained with the Siemens SOMATOM CT scanner (Siemens, Erlangen, Germany) approximately one month after the seed implant to allow the decrease of prostate swelling. The slice thickness of the CT scan was $1 \mathrm{~mm}$ for the purpose of clear delineation of each radioactive seed. The quality of the PSI was evaluated based on these CT scans.

\subsection{Treatment Planning}

Both IMPT and IMRT plans were created based on the post-implant CT scans. The prostate and seminal vesicles 
were contoured as the clinical target volume (CTV). The planning target volume (PTV) was created with a margin of $5 \mathrm{~mm}$ around the CTV except that 3-mm margin was used at the prostate rectal interface to reduce the risk of rectal morbidity. Other critical organs such as bladder, rectum, penile bulb, femoral heads and urethra were also contoured. The urethra was defined as a 5-mm diameter volume using a surrogate technique as previously described [11]. The PTV coverage in plan optimization was such that $100 \%$ of the prescribed dose was required to cover at least $98 \%$ of the PTV. Rectum and bladder were spared as much as possible without compromising PTV coverage. The calculation grids were set at $1 \mathrm{~mm}$ and planning priority was given to PTV coverage. To accommodate the high density of I-125 radioactive seeds, the CT number to electron density table and CT number to relative stopping power table were both extended to CT number up to 6000 with linear extrapolation.

Proton treatment plans were created using intensity modulated proton beams from a Varian proton cyclotron accelerator with maximum energy of $250 \mathrm{MeV}$. Two parallel opposed lateral beams were generated in the Eclipse treatment planning system (RTP) (Varian Medical System, Palo Alto, CA, Varian version 10.0). The proton dose calculation was based on the convolution superposition algorithm with dose Kernels derived from Monte Carlo Calculation. The prescription dose was 45 Gray-equivalents (GyE) in 1.8-GyE fractions with an RBE of 1.1. IMRT boost plans with the same prescription dose and similar dose constraints were generated with seven co-planner $6 \mathrm{MV}$ photon beams of a Trilogy Linac in the Eclipse RTP software Version 10.0 (Varian Medical System, Palo Alto, CA). Several planning-purpose structures were generated to assist the plan optimization to avoid hot spots and improve OARs sparing, including 1) Body minus PTV 0.5 cm expansion; 2) Rectum minus PTV; 3) Bladder minus PTV. Typical dose constraints are listed in Table 1. For comparison purpose, IMRT plans were also normalized to have $98 \%$ of the PTV volume covered by $100 \%$ of the prescribed dose.

\subsection{Plan Evaluation}

To compare the dose coverage between IMPT and IMRT plans, dose-volume histograms (DVH) were generated for quantitative evaluations. Specifically, the following parameters were compared between these two sets of plans:

- PTV coverage: $\mathrm{D}_{1 \%}$ (minimum dose delivered to 1\% of PTV) and $\mathrm{D}_{99 \%}$ (minimum dose delivered to $99 \%$ of PTV) were used as surrogate markers for maximum and minimum doses within PTV.

- Conformity Index (CI) was used to evaluate the dose conformity of the two sets of plans compared to prescribed dose. The CI is defined as

$$
\mathrm{CI}=\left(V_{P T V} * V_{T V}\right) /\left(T V_{P V}\right)^{2}
$$

where $V_{P T V}$ is the PTV volume (cc), $V_{T V}$ is the body volume that is covered by the prescribed isodose line, and $T V_{P V}$ is the PTV volume covered by the prescribed dose. In the ideal case, $V_{P T V}=V_{T V}=T V_{P V}$ and CI $=1[12]$ [13].

- Homogeneity Index (HI) was used to assess the dose uniformity and is defined as the ratio of $\mathrm{D}_{5 \%}$ to $\mathrm{D}_{95 \%}$ in the PTV, where $\mathrm{D}_{5 \%}$ is the maximum dose delivered to $5 \%$ of the PTV and $\mathrm{D}_{95 \%}$ is the minimum dose delivered to $95 \%$ of the PTV. HI is always equal or greater than 1. Ideally when PTV is uniformly covered with a certain isodose line, HI equals to 1.0.

- Organs at Risk (OARs), which included rectum, bladder, urethra and femoral heads. Dose distributions to these surrounding normal tissues were compared for both IMPT and IMRT plans.

- Integral Dose, which is defined as the total dose received by the body volume in the CT scan minus the PTV volume.

Paired T test was performed to analyze the difference between IMPT and IMRT boost plans with p value less than 0.05 indicating statistical significance. SAS (version 9.2, SAS Institute, Cary, NC) statistical software was utilized for all the statistical analysis.

\section{Results}

\section{Comparison of Dose Distributions between IMPT and IMRT Plans}

The typical difference of dose distribution between IMPT and IMRT plans were illustrated in Figure 1 and Figure 2 for one of the patients in the study. The most striking difference was the much larger irradiated volume in the IMRT plan compared to IMPT. It was also obvious from Figure 1 that the low dose distribution to the rectum was significantly smaller in IMPT plans. 
Table 1. Typical IMPT optimization criteria.

\begin{tabular}{ccccc}
\hline \multirow{2}{*}{ Optimization } & \multicolumn{2}{c}{ Volume (\%) } & Dose (\% of Dx) & Priority \\
\hline \multirow{2}{*}{ PTV } & Upper & 0 & $108 \%$ & 150 \\
\multirow{2}{*}{ Body } & Lower & 100 & $104 \%$ & 150 \\
& Upper & 0 & $110 \%$ & 350 \\
\multirow{2}{*}{ Bladder-PTV } & Upper & 100 & $0 \%$ & 50 \\
& Upper & 40 & $25 \%$ & 50 \\
& Upper & 0 & $60 \%$ & 50 \\
Rectum-PTV & Upper & 100 & $0 \%$ & 50 \\
& Upper & 35 & $8 \%$ & 50 \\
Body-PTV-0.5 cm & Upper & 0 & $70 \%$ & 50 \\
\hline
\end{tabular}

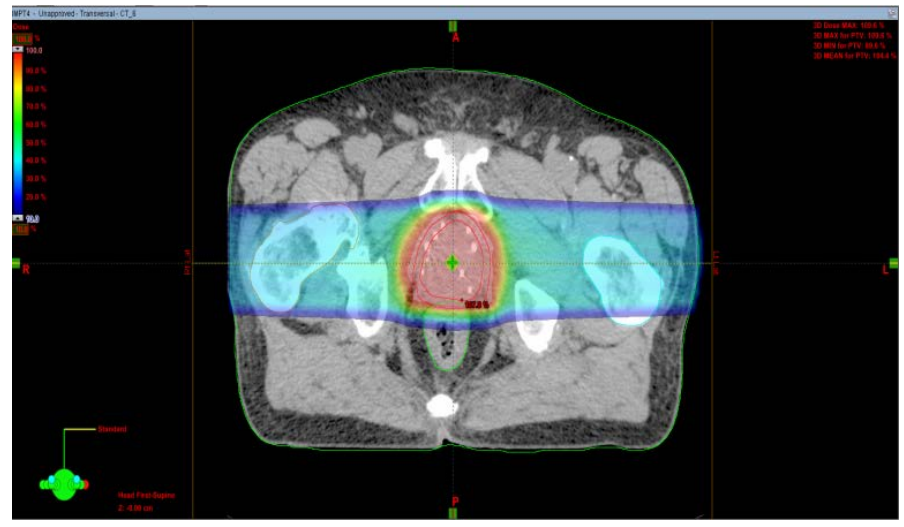

Figure 1. A representative IMPT plan.

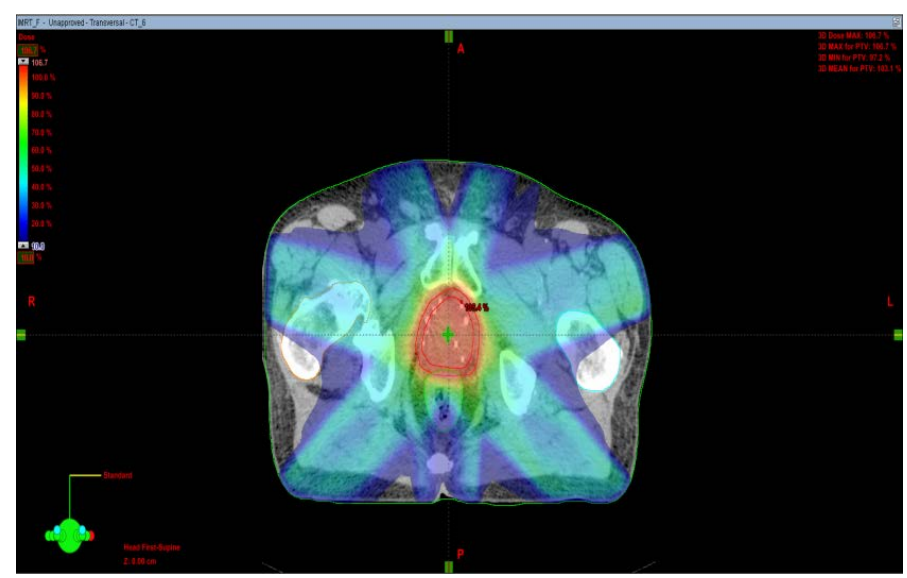

Figure 2. A representative IMRT plan.

Figure 3 compared the DVHs of PTV and some Organ at Risk (OARs) between the IMRT and IMPT plan for the same patient.

\section{PTV and Prostate Coverage}

For the ten patients selected in this study, both IMRT and IMPT plans demonstrated excellent dosimetric coverage for PTV volumes. On average, $99.91 \%$ and $99.84 \%$ of the PTV volumes were covered by $95 \%$ of the prescribed dose for IMRT and IMPT plans, respectively. The dosimetric comparison of the PTV coverage between IMPT and IMRT plans for all patients were summarized in Table 1. The average value of CI for the ten 
IMPT plans was estimated to be 1.14, while the corresponding average CI for the IMRT plans was 1.10 (p value $=0.002$ ). On the other hand, IMPT plans demonstrated slightly better homogeneity with an average HI of 1.04 compared to 1.05 for IMRT plans ( $p$ value $=0.04$ ). No significant difference was observed in terms of the $\mathrm{D}_{99 \%}$ and $\mathrm{D}_{1 \%}$ values between IMPT and IMRT plans since $\mathrm{p}$ values were greater than 0.05 .

\section{Rectum}

Compared to conventional IMRT boost plans, IMPT boost plans demonstrated improved sparing of rectum, while providing similar PTV coverage. The mean rectal dose in IMRT plans (21.0\%) was significantly greater than that in IMPT plans (12.8\%) with $\mathrm{p}<0.05$. The rectum $\mathrm{D}_{15 \%}, \mathrm{D}_{25 \%}, \mathrm{D}_{35 \%}$ and $\mathrm{D}_{50 \%}$ with IMPT plans were $31.2 \%, 13.7 \%, 5.9 \%$ and $1.6 \%$ respectively, compared to $44.1 \%, 28.2 \%, 18.1 \%$ and $11.4 \%$ respectively for the IMRT plans (all p values $<0.05$ ). The $\mathrm{D}_{2 c c}$ values for the IMPT plans were not significantly different from those of the IMRT plans ( $p$ value $=0.643$ ). A detailed comparison for rectum between IMPT and IMRT was listed in Table 2.

\section{Bladder}

IMPT also showed better bladder sparing. The mean dose of bladder decreased from $22.7 \%$ for IMRT plan to $18.2 \%$ (IMPT) $(\mathrm{p}<0.05)$ of the prescribed dose. The bladder $\mathrm{D}_{35 \%}$ and $\mathrm{D}_{50 \%}$ with IMPT plans were $12.8 \%$ and $4.3 \%$ respectively, which were lower than $21.3 \%$ and $12.2 \%$ with IMRT plans (both p values $<0.05$ ). $D_{15 \%}, D_{25 \%}$ and $\mathrm{D}_{2 \text { cc }}$ were not significantly different between IMPT and IMRT plans (All p value $>0.05$ ). The detailed comparison for bladder between IMPT and IMRT was listed in Table 3.

\section{Urethra}

No significant difference was observed for urethra dose between IMPT and IMRT plans. The mean dose of urethra in IMPT plans and IMRT plans were $104 \%$ to $103 \%$ of the prescribed dose respectively (p value $=0.06$ ). Table 4 illustrated the detailed comparison of urethra and other normal tissues between IMPT and IMRT plans.

\section{Pelvis (Integral Dose)}

One of the drawbacks of IMRT is that larger volume of patient body may receive some radiation dose, which may potentially cause secondary cancer [14]. It was noted that the volume of pelvis receiving radiation doses was significantly reduced in IMPT plans (Table 5) compared to IMRT plans. The integral dose comparison was evaluated by the mean dose of body volume outside the PTV, which was also named "Body-PTV". The mean doses to the volume of "Body-PTV" were estimated as $5.8 \%$ and $17.4 \%$ of the prescribed dose for IMPT plans and IMRT plans respectively (p value $<0.0001$ ), proving much higher dose drop off of IMPT plans compared to IMRT plans.

\section{Femoral Heads}

It was found that the $\mathrm{D}_{5 \%}$ of femoral heads comparison favored IMPT plans since the average $\mathrm{D}_{5 \%}$ dose of IMPT plans was $40.2 \%$ of prescribed dose compared to $55.2 \%$ for IMRT plans.

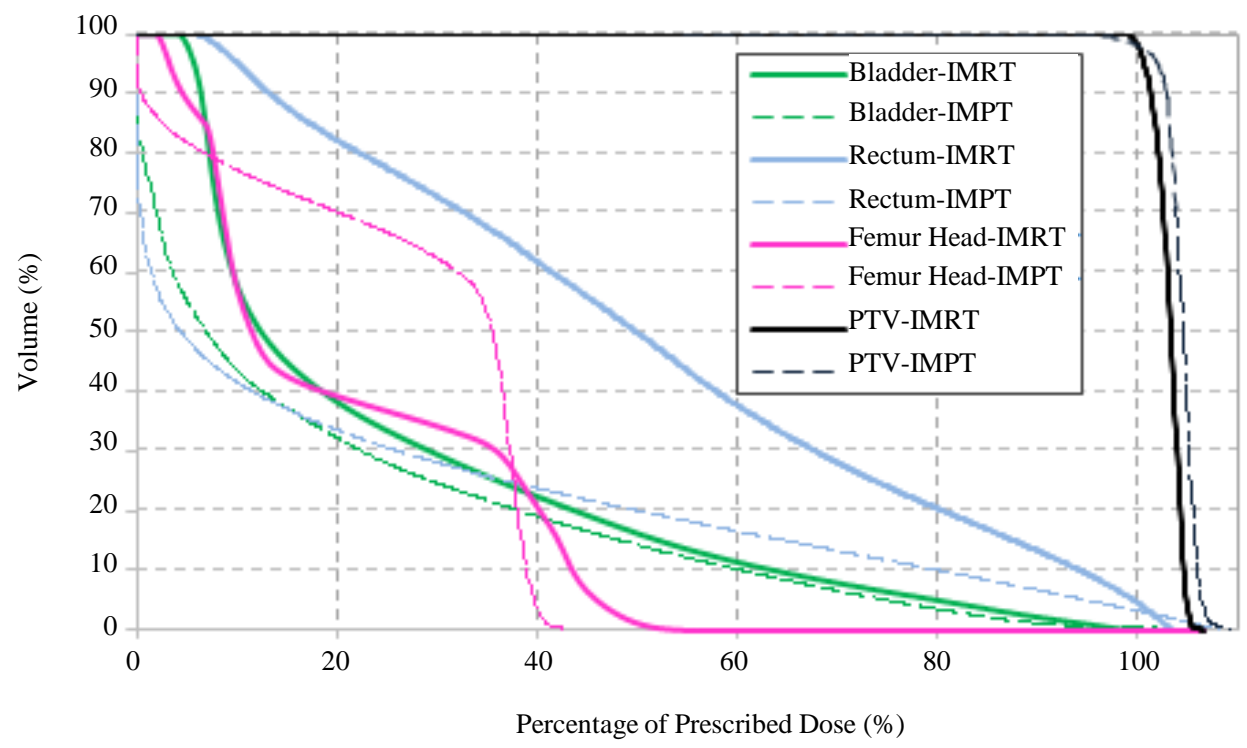

Figure 3. Dose volume histogram of plan comparison between IMPT plan and IMRT plan. 
Table 2. PTV coverage comparison between IMPT and IMRT.

\begin{tabular}{cccccccccc}
\hline Patients & \multicolumn{3}{c}{ IMRT } & \multicolumn{3}{c}{ IMPT } \\
\hline PTV & D $_{5 \%}$ & D $_{95}$ & CI & HI & D $_{5 \%}$ & D $_{95 \%}$ & CI & HI \\
\hline P1 & 4766 & 4546 & 1.15 & 1.04 & 4742 & 4578 & 1.15 & 1.04 \\
P2 & 4683 & 4518 & 1.07 & 1.04 & 4711 & 4555 & 1.13 & 1.03 \\
P3 & 4781 & 4557 & 1.12 & 1.05 & 4727 & 4569 & 1.13 & 1.03 \\
P4 & 4702 & 4525 & 1.07 & 1.04 & 4784 & 4577 & 1.15 & 1.05 \\
P5 & 4748 & 4544 & 1.12 & 1.05 & 4785 & 4612 & 1.18 & 1.04 \\
P6 & 4719 & 4530 & 1.09 & 1.04 & 4717 & 4555 & 1.11 & 1.04 \\
P7 & 4694 & 4520 & 1.07 & 1.04 & 4780 & 4582 & 1.16 & 1.04 \\
P8 & 4763 & 4443 & 1.10 & 1.07 & 4766 & 4576 & 1.15 & 1.04 \\
P9 & 4709 & 4525 & 1.10 & 1.04 & 4732 & 4564 & 1.14 & 1.04 \\
P10 & 4711 & 4533 & 1.09 & 1.04 & 4674 & 4540 & 1.10 & 1.03 \\
Average & 4728 & 4524 & 1.10 & 1.05 & 4742 & 4571 & 1.14 & 1.04 \\
P-value & 0.372 & 0.003 & 0.002 & 0.044 & 0.372 & 0.003 & 0.002 & 0.044 \\
\hline
\end{tabular}

Note: CI = Conformity Index; HI = Homogeneity Index.

Table 3. Rectum dose comparison between IMPT and IMRT.

\begin{tabular}{|c|c|c|c|c|c|}
\hline \multirow{2}{*}{$\begin{array}{l}\text { Rectum } \\
\text { Metric }\end{array}$} & \multicolumn{2}{|c|}{ IMRT } & \multicolumn{2}{|c|}{ IMPT } & \multirow{2}{*}{$\mathrm{p}$ value } \\
\hline & Average & STDEV & Average & STDEV & \\
\hline Dmean (Gy/CGE) & $21.0 \%$ & $5.0 \%$ & $12.8 \%$ & $6.1 \%$ & $<0.0001$ \\
\hline D50\% & $11.4 \%$ & $4.2 \%$ & $1.6 \%$ & $3.1 \%$ & $<0.0001$ \\
\hline D35\% & $18.1 \%$ & $5.3 \%$ & $5.9 \%$ & $8.1 \%$ & $<0.0001$ \\
\hline D25\% & $28.2 \%$ & $6.4 \%$ & $13.7 \%$ & $13.8 \%$ & 0.0007 \\
\hline D15\% & $44.1 \%$ & $9.6 \%$ & $31.2 \%$ & $19.7 \%$ & 0.0141 \\
\hline $\mathrm{D} 2 \mathrm{cc}$ & $77.8 \%$ & $15.3 \%$ & $79.3 \%$ & $15.5 \%$ & 0.6435 \\
\hline
\end{tabular}

Table 4. Bladder dose comparison between IMPT and IMRT.

\begin{tabular}{|c|c|c|c|c|c|}
\hline \multirow{2}{*}{$\begin{array}{c}\text { Bladder } \\
\text { Metric }\end{array}$} & \multicolumn{2}{|c|}{ IMRT } & \multicolumn{2}{|c|}{ IMPT } & \multirow{2}{*}{$\mathrm{p}$ value } \\
\hline & Average & STDEV & Average & STDEV & \\
\hline $\mathrm{D}_{\text {mean }}(\mathrm{Gy} / \mathrm{CGE})$ & $22.7 \%$ & $8.2 \%$ & $18.2 \%$ & $10.0 \%$ & $<0.0001$ \\
\hline $\mathrm{D}_{50 \%}$ & $12.2 \%$ & $7.3 \%$ & $4.3 \%$ & $8.2 \%$ & $<0.0001$ \\
\hline $\mathrm{D}_{35 \%}$ & $21.3 \%$ & $12.3 \%$ & $12.8 \%$ & $18.5 \%$ & $<0.0001$ \\
\hline $\mathrm{D}_{25 \%}$ & $31.2 \%$ & $17.0 \%$ & $23.8 \%$ & $25.3 \%$ & 0.0007 \\
\hline $\mathrm{D}_{15 \%}$ & $46.7 \%$ & $20.7 \%$ & $46.0 \%$ & $28.5 \%$ & 0.0141 \\
\hline $\mathrm{D}_{2 \mathrm{cc}}$ & $96.2 \%$ & $13.1 \%$ & $98.9 \%$ & $10.8 \%$ & 0.6435 \\
\hline
\end{tabular}

Table 5. Normal tissue dose comparison between IMPT and IMRT.

\begin{tabular}{|c|c|c|c|c|c|c|}
\hline \multirow{2}{*}{ Volume } & \multirow{2}{*}{ Metric } & \multirow{2}{*}{$\begin{array}{c}\text { IMRT } \\
\text { Average }\end{array}$} & \multicolumn{3}{|c|}{ IMPT } & \multirow{2}{*}{$\mathrm{p}$ value } \\
\hline & & & STDEV & Average & STDEV & \\
\hline \multicolumn{7}{|l|}{ Urethra } \\
\hline & $\mathrm{D}_{\text {mean }}(\mathrm{Gy} / \mathrm{CGE})$ & $103.0 \%$ & $1.9 \%$ & $104.0 \%$ & $1.2 \%$ & 0.06 \\
\hline \multicolumn{7}{|c|}{ Femoral Heads } \\
\hline & $\mathrm{D}_{5 \%}$ & $55.2 \%$ & $8.2 \%$ & $40.2 \%$ & $1.1 \%$ & 0.0003 \\
\hline \multicolumn{7}{|c|}{ Body minus PTV } \\
\hline & $\mathrm{D}_{\text {mean }}(\mathrm{Gy} / \mathrm{CGE})$ & $17.4 \%$ & $6.0 \%$ & $5.8 \%$ & $1.9 \%$ & $<0.0001$ \\
\hline
\end{tabular}




\section{Discussion}

In this study, PSI with an IMPT boost proved to be a valuable option for prostate cancer patients. In combination with PSI, IMPT can deliver a significantly higher dose to gross disease to increase the likelihood of tumor control. Compared to IMRT boost plans, IMPT was able to deliver a comparable or better treatment in terms of normal tissue sparing.

The high density of implanted seeds has been a concern for IMPT as boost treatment. Even though it has been shown that high density markers could cause dose perturbation [15]-[17], the effect is only limited to the areas around the markers. In the case of prostate seed implants, the under-dose area around seeds in a proton beam is not expected to be dosimetrically significant in overall dose delivery because the extremely high dose in the vicinity of radioactive seeds is more than enough to compensate the under dose in the shadows of the seeds in a proton beam. Analysis of the 10 seed implant cases showed that the mean dose is $199 \%$ of the prescribed dose at $2 \mathrm{~mm}$ away from each individual seed. The mean dose rate at $5 \mathrm{~mm}$ away from the seeds is $33 \%$ higher than the prescription dose. The biological equivalent dose (BED) is even higher in the immediate vicinity of the seeds. Therefore, affected dose coverage area was minimum and negligible considering the higher dose from seed implant.

It is also worthy to mention that a linear extrapolation of CT-relative stopping power curve (CT-RSP) was applied in this study. There have been plenty of literatures [18]-[20] exploring the importance of CT-RSP in the accuracy of proton dose calculation. However, for the same reason mentioned previously, the dose contribution to the region around the seed is extremely high from the implanted radioactive seeds. The high $\mathrm{Z}$ material of the seeds has very little effect in the combined overall dose distribution even though there may be under-dose region or certain inaccuracy of dose calculation around each seed in a proton beam.

Radiobiologically, a single rounded-off value of RBE of 1.1 for proton relative to megavoltage photon beams has been used in most proton treatment facilities. In this study, the comparison was mainly concerned with the relative dose distributions between IMPT and IMRT plans, which eliminated the issue of radiobiological dose summation and radiobiological difference. The analysis of BED difference between the LDR brachytherapy treatment and proton beam was beyond the scope of this study but could be solved by radiobiological planning in the future. Some commercial software, such as MIM Vista (Cleveland, Ohio), is capable to perform deformable dose evaluation and summation of Brachytherapy plans and EBRT plans using the built-in BED models.

In addition, only two laterally opposed proton beams for IMPT planning was investigated in this study. Further studies could be performed with different gantry angles and multiple beams for better optimization. It could improve the CI, HI and normal tissues sparing at the expense of the setup and delivery time. For patients with hip replacement, IMPT treatment with other gantry angle combination is also necessary due to hip prostheses effect.

\section{Conclusion}

Prostate Seed Implant (PSI) with an Intensity Modulation Proton Therapy (IMPT) boost can be a valuable option for prostate cancer patients. The IMPT boost delivers comparable or better treatment in terms of normal tissue sparing compared to IMRT plans. Future research will be focused on the implementation of IMPT on other types of cancer diseases.

\section{Acknowledgements}

The authors would like to acknowledge Xiaohe Yu for his assistance in this work.

\section{References}

[1] Jani, A.B., Hand, C.M., Lujan, A.E., Roeske, J.C., Zagaja, G.P., Vijayakumar, S. and Pelizzari, C.A. (2004) Biological Effective Dose for Comparison and Combination of External Beam and Low-Dose Rate Interstitial Brachytherapy Prostate Cancer Treatment Plans. Medical Dosimetry, 29, 42-48. http://dx.doi.org/10.1016/j.meddos.2003.09.005

[2] Zaider, M., Zelefsky, M.J., Cohen, G.N., Chui, C.-S., Yorke, E.D., Ben-Porat, L. and Happersett, L. (2005) Methodology for Biologically-Based Treatment Planning for Combined Low-Dose-Rate (Permanent Implant) and High-DoseRate (Fractionated) Treatment of Prostate Cancer. International Journal of Radiation Oncology*Biology*Physics, 61, 702-713. http://dx.doi.org/10.1016/j.ijrobp.2004.06.251 
[3] Zelefsky, M.J., Nedelka, M.A., Arican, Z.-L., Yamada, Y., Cohen, G.N., Shippy, A.M., Park, J.J. and Zaider, M. (2008) Combined Brachytherapy with External Beam Radiotherapy for Localized Prostate Cancer: Reduced Morbidity with an Intraoperative Brachytherapy Planning Technique and Supplemental Intensity-Modulated Radiation Therapy. Brachytherapy, 7, 1-6. http://dx.doi.org/10.1016/j.brachy.2007.12.002

[4] Zilli, T., Boudreau, C., Filion, E.J., Donath, D. and Taussky, D. (2011) Combined Intensity-Modulated Radiation Therapy vs. Three-Dimensional Highly Conformal Radiotherapy after ${ }^{125}$ I Prostate Permanent Seed Brachytherapy: A Comparative Treatment Planning Study. Brachytherapy, 10, 416-420. http://dx.doi.org/10.1016/j.brachy.2010.12.005

[5] Cao, M., Ko, S.-C., Slessinger, E.D., DesRosiers, C.M., Johnstone, P.A. and Das, I.J. (2011) A Simple Method for Dose Fusion from Multimodality Treatment of Prostate Cancer: Brachytherapy to External Beam Therapy. Brachytherapy, 10, 214-220. http://dx.doi.org/10.1016/j.brachy.2010.08.007

[6] Albert, M., Tempany, C.M., Schultz, D., Chen, M.-H., Cormack, R.A., Kumar, S., et al. (2003) Late Genitourinary and Gastrointestinal Toxicity after Magnetic Resonance Image-Guided Prostate Brachytherapy with or without Neoadjuvant External Beam Radiation Therapy. Cancer, 98, 949-954. http://dx.doi.org/10.1002/cncr.11595

[7] Sarosdy, M.F. (2004) Urinary and Rectal Complications of Contemporary Permanent Transperineal Brachytherapy for Prostate Carcinoma with or without External Beam Radiation Therapy. Cancer, 101, 754-760. http://dx.doi.org/10.1002/cncr.20446

[8] Zeitlin, S.I., Sherman, J., Raboy, A., Lederman, G. and Albert, P. (1998) High Dose Combination Radiotherapy for the Treatment of Localized Prostate Cancer. The Journal of Urology, 160, 91-95; discussion 95-96. http://dx.doi.org/10.1016/S0022-5347(01)63042-8

[9] Lomax, A.J., Boehringer, T., Coray, A., Egger, E., Goitein, G., Grossmann, M., et al. (2001) Intensity Modulated Proton Therapy: A Clinical Example. Medical Physics, 28, 317-324. http://dx.doi.org/10.1118/1.1350587

[10] Polo, A., Salembier, C., Venselaar, J. and Hoskin, P. (2010) Review of Intraoperative Imaging and Planning Techniques in Permanent Seed Prostate Brachytherapy. Radiotherapy and Oncology, 94, 12-23. http://dx.doi.org/10.1016/j.radonc.2009.12.012

[11] Bucci, J., Spadinger, I., Hilts, M., Sidhu, S., Smith, C., Keyes, M. and James Morris, W. (2002) Urethral and Periurethral Dosimetry in Prostate Brachytherapy: Is There a Convenient Surrogate? International Journal of Radiation Oncology*Biology*Physics, 54, 1235-1242. http://dx.doi.org/10.1016/S0360-3016(02)03054-7

[12] Nakamura, J.L., Verhey, L.J., Smith, V., Petti, P.L., Lamborn, K.R., Larson, D.A., et al. (2001) Dose Conformity of Gamma Knife Radiosurgery and Risk Factors for Complications. International Journal of Radiation Oncology*Biology*Physics, 51, 1313-1319. http://dx.doi.org/10.1016/S0360-3016(01)01757-6

[13] Paddick, I. (2000) A Simple Scoring Ratio to Index the Conformity of Radiosurgical Treatment Plans. Technical Note. Journal of Neurosurgery, 93, 219-222.

[14] Hall, E.J. and Wuu, C.S. (2003) Radiation-Induced Second Cancers: The Impact of 3D-CRT and IMRT. International Journal of Radiation Oncology*Biology*Physics, 56, 83-88. http://dx.doi.org/10.1016/S0360-3016(03)00073-7

[15] Newhauser, W., Fontenot, J., Koch, N., Dong, L., Lee, A., Zheng, Y.S., et al. (2007) Monte Carlo Simulations of the Dosimetric Impact of Radiopaque Fiducial Markers for Proton Radiotherapy of the Prostate. Physics in Medicine and Biology, 52, 2937-2952. http://dx.doi.org/10.1088/0031-9155/52/11/001

[16] Newhauser, W.D., Koch, N.C., Fontenot, J.D., Rosenthal, S.J., Gombos, D.S., Fitzek, M.M. and Mohan, R. (2007) Dosimetric Impact of Tantalum Markers Used in the Treatment of Uveal Melanoma with Proton Beam Therapy. Physics in Medicine and Biology, 52, 3979-3790. http://dx.doi.org/10.1088/0031-9155/52/13/021

[17] Mendenhall, N.P., Malyapa, R.S., Su, Z., Yeung, D., Mendenhall, W.M. and Li, Z.F. (2011) Proton Therapy for Head and Neck Cancer: Rationale, Potential Indications, Practical Considerations, and Current Clinical Evidence. Acta Oncologica, 50, 763-771. http://dx.doi.org/10.3109/0284186X.2011.590147

[18] Yohannes, I., Kolditz, D., Langner, O. and Kalender, W.A. (2012) A Formulation of Tissue- and Water-Equivalent Materials Using the Stoichiometric Analysis Method for CT-Number Calibration in Radiotherapy Treatment Planning. Physics in Medicine and Biology, 57, 1173-1190. http://dx.doi.org/10.1088/0031-9155/57/5/1173

[19] Schaffner, B. and Pedroni, E. (1998) The Precision of Proton Range Calculations in Proton Radiotherapy Treatment Planning: Experimental Verification of the Relation between CT-HU and Proton Stopping Power. Physics in Medicine and Biology, 43, 1579-1592. http://dx.doi.org/10.1088/0031-9155/43/6/016

[20] Cheng, C.-W., Zhao, L., Wolanski, M., Zhao, Q.Y., James, J., Dikeman, K., et al. (2013) Comparison of Tissue Characterization Curves for Different CT Scanners: Implication in Proton Therapy Treatment Planning. Translational Cancer Research, 1, 236-246. 
Scientific Research Publishing (SCIRP) is one of the largest Open Access journal publishers. It is currently publishing more than 200 open access, online, peer-reviewed journals covering a wide range of academic disciplines. SCIRP serves the worldwide academic communities and contributes to the progress and application of science with its publication.

Other selected journals from SCIRP are listed as below. Submit your manuscript to us via either submit@scirp.org or Online Submission Portal.
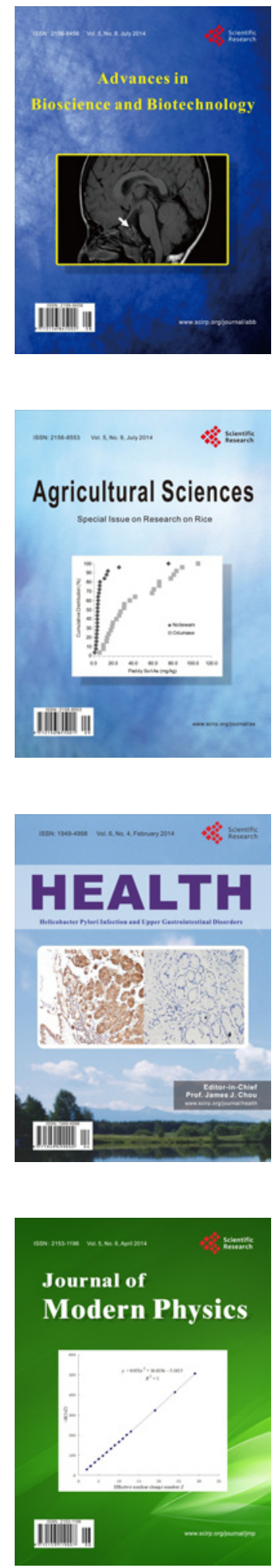
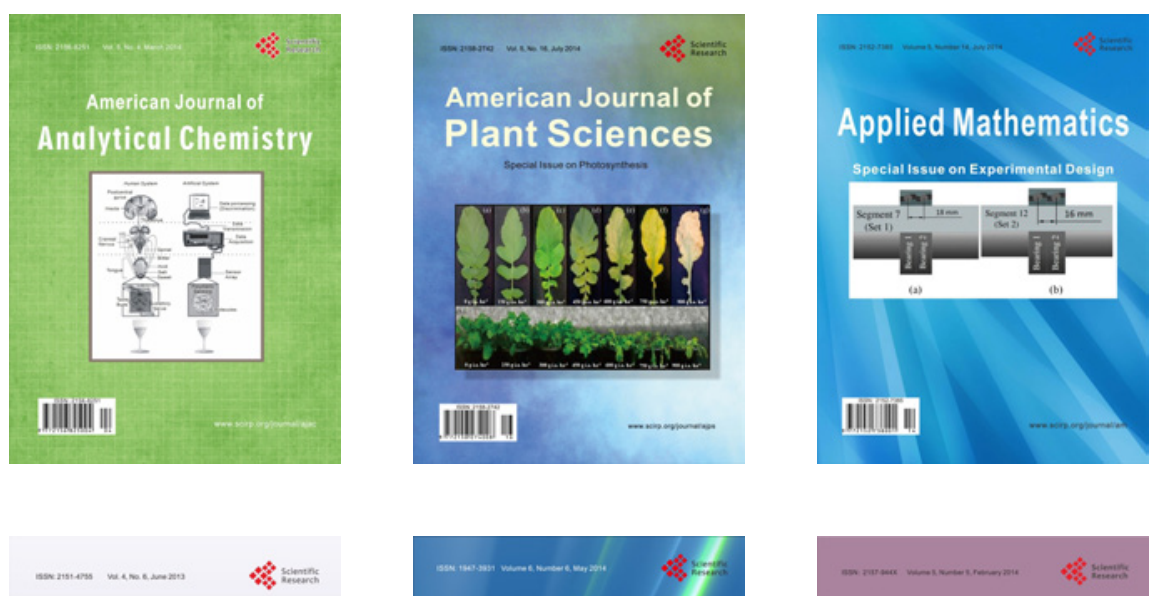

Creative Education
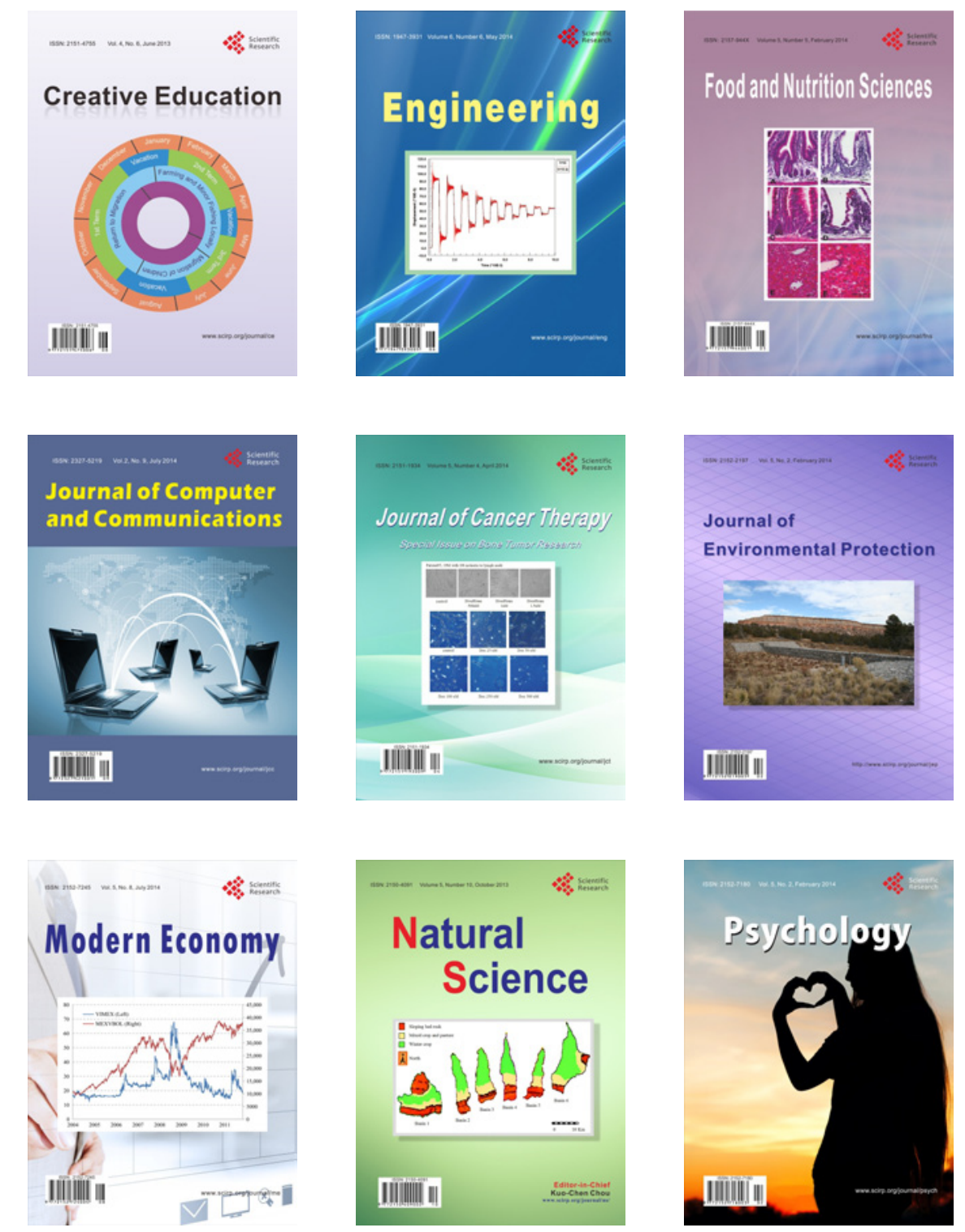